\title{
General Psychiatry Research progress and debates on gaming disorder
}

To cite: Wang Q, Ren H, Long J, et al. Research progress and debates on gaming disorder. General Psychiatry 2019;32:e100071. doi:10.1136/ gpsych-2019-100071

Received 02 April 2019 Revised 28 May 2019 Accepted 03 June 2019
Check for updates

(C) Author(s) (or their employer(s)) 2019. Re-use permitted under CC BY-NC. No commercial re-use. See rights and permissions. Published by BMJ.

Department of Behavioral Medicine, The Second Xiangya Hospital of Central South University, Changsha, China

Correspondence to Dr Tieqiao Liu; liutieqia0123@csu.edu.cn

Qianjin Wang, Honghong Ren, Jiang Long, Yueheng Liu, Tieqiao Liu

\section{ABSTRACT}

Gaming disorder has become a significant issue in mental healthcare. While gaming is an important form of entertainment, excessive gaming may cause serious consequences for players. At present, there are still controversies in the academic community concerning the public health problems related to gaming disorder. This article attempts to expound the definition, epidemiology, aetiology, diagnosis, treatment and prevention of gaming disorder, in order to contribute to future conceptualization of gaming disorder.

\section{DEFINITION AND PREVALENCE OF GAMING DISORDER}

Gaming disorder is defined as a negative gaming behaviour pattern characterised by the loss of control over the game and total time spent playing the game, resulting in other interests and daily activities being given away to the game. Even if there are negative consequences, game behaviour continues or continues to escalate. For the diagnosis of gaming disorder, the game behaviour pattern must be serious enough for at least 12 months to cause significant damage in an individual's personal life, family, social, educational, professional, or other important areas of functioning. ${ }^{1}$

'The statistical report on the development of the internet in China' pointed out that China had 486 million people playing internet games by June 2018, accounting for $60.6 \%$ of all internet users. ${ }^{2}$ A recent systematic review of epidemiological studies on gaming disorder found that the prevalence of gaming disorder was $0.7 \%-27.5 \%$, and it was mainly among young men. ${ }^{3}$ Another group conducted a meta-analysis of 36 studies in China, covering 362328 internet gamers. ${ }^{4}$ The prevalence of online gaming disorder in China was $3.5 \%-17 \%$. The prevalences of gaming disorder in Europe and the USA were relatively low, for example, the USA was about $0.3 \%-1.0 \%,{ }^{5}$ and Germany was $1.16 \%{ }^{6}$

\section{CAUSES AND POSSIBLE MECHANISMS OF GAMING DISORDER}

The cause of gaming disorder is not yet fully understood. Most research indicates that the following aspects may be involved: first, the game's built-in reward system may be the cause of the gaming disorder. ${ }^{7}$ For example, many games, particularly massively multiplayer online role-playing games, rely on a 'compulsion loop', a cycle of activities that involve rewarding the player and driving them to continue through another cycle, retaining them in the game. ${ }^{8}$ Many players refuse to quit the game because they are rewarded in the game. The expectation of this kind of reward can increase dopamine in the brain, activate the reward system and, once the player is rewarded, he/she can become addicted in the long run. This mechanism is similar to the neurobiological mechanism of gambling disorder. ${ }^{9}$ Moreover, in the virtual world generated by the game, the person with gaming disorder can gain confidence and satisfaction that cannot be obtained in the real world. ${ }^{4}$ In addition, high testosterone load may be a risk factor for adults with gaming disorder. ${ }^{10}$ There is research indicating that ${ }^{411}$ genetic factors, marital status, history of abuse and trauma, modes of education, history of mental disorders, demographic factors, personality and psychological factors, family and social factors, and factors related to the game (such as game type and game experience) also play an important role in gaming disorder.

\section{DIAGNOSTICS OF GAMING DISORDER}

There is still no consensus on the diagnostic criteria for gaming disorder. Many of the previously proposed criteria for gaming disorder were similar to the fourth edition of the Diagnostic and Statistical Manual of Mental Disorders' (DSM-IV) diagnoses for substance use disorders, which relied on scales and questionnaires. ${ }^{12}$

In May 2013, the DSM-5 issued by the American Psychiatric Association did not include gaming disorder. It was believed that there was not enough evidence to classify it as a mental disorder, but the proposed standard 
for gaming disorder was placed in the appendix of DSM-5 as a 'clinical phenomenon requiring further research'. ${ }^{13}$ The revision experts of DSM-5 admitted that gaming disorder would have a negative impact on patients' personal and social lives. Therefore, they still provided nine diagnostic criteria for gaming disorder, and believed that meeting five of the nine criteria over the course of 12 months warranted a diagnosis of gaming disorder. ${ }^{3}$ Those nine criteria were the following: (1) being completely focused on the game; (2) when stopping the game, symptoms such as anxiety and irritability appear; (3) the time spent playing games gradually increases; (4) those with gaming disorder are unable to reduce the amount of time spent playing the game and cannot quit the game; (5) individuals with gaming disorder will give up other activities and lose interest in other hobbies; (6) even when the person understands that the game has a negative impact on life, they still focus on the game; (7) the person will hide the amount of playing time from family members or others; (8) mitigating negative emotions such as guilt, despair, and so forth, due to playing games will be present; and (9) loss of functioning in work, studies or social life due to gaming. It should be pointed out that only DSM-5 has given diagnostic criteria for online gaming disorder.

In June 2018, the WHO included gaming disorder in the chapter of substance and behavioural addiction in the 11th edition of the International Classification of Diseases and Related Health Problems (ICD-11). They listed the diagnostic criteria as the following: (1) obsession with the game that is difficult to control for more than 12 months; (2) the degree of obsession with the game is higher than other interests, resulting in a reduction in daily activities; and (3) even if one is aware of the negative impact, the game behaviour continues or escalates. ${ }^{14}$ However, the WHO's move has caused opposition from some scholars and members of the Game Association. They believe that the classification of gaming disorder lacks scientific basis, and it is not yet clear whether gaming disorder is caused by game activities themselves or is affected by other diseases. Such a diagnosis may cause discrimination for many game players. ${ }^{15}$ At present, there are many uncertainties about gaming disorder in many ways, ${ }^{16}$ which may mistake the normal players for those with gaming disorder, and therefore may cause excessive diagnosis and treatment.

At present, there are both similarities and differences in the two diagnostic systems for gaming disorder. ${ }^{17}$ The same points all emphasise the uncontrolled reckless game behaviour within 12 months, which has caused serious consequences in one's personal and social life, and so on. Despite the similar psychological craving, tolerance and withdrawal symptoms to the substance use disorder, the individuals will continue their excessive playing of the game. The differences are: (1) ICD-11 incorporates gaming disorder into the substance and behavioural disorder chapter, including all game forms such as online games, offline games, or other unspecified games. DSM- 5 incorporates gaming disorder into the chapter on clinical phenomena that require further research, emphasising only online games. (2) ICD-11 contains the diagnostic classification of hazardous use of games, but this diagnostic classification is not seen in DSM-5. (3) ICD-11 is a diagnostic guide. It introduces the diagnosis and differential diagnosis of gaming disorder and provides good guidance for diagnosis. DSM- 5 is a diagnostic standard. One can be diagnosed with a gaming disorder by meeting five or more of the nine criteria. Furthermore, the diagnostic criteria of the DSM- 5 are more detailed than the ICD-11, so it has good operability. (4) DSM-5 also discusses the prevalence, diagnosis, influencing factors, differential diagnosis and comorbidity of online gaming disorder.

\section{TREATMENT OF GAMING DISORDER}

Excessive gaming can damage an individual's daily life and social functioning. Therefore, professional treatment for people with gaming disorder is necessary. Unfortunately, there is currently a lack of recognised treatment measures for gaming disorder. ${ }^{18}$ Because the pathogenesis of the gaming disorder is not yet clear, the current intervention measures are basically based on the treatment experience of mental disorders such as substance use disorder. Treatment measures generally include psychobehavioural treatment, drug treatment and comprehensive treatment. ${ }^{19}$

\section{Psychological therapy}

Psychological therapy, including individual and group therapy, is currently the most commonly used method for treating gaming disorder.

\section{Individual treatment}

Among individual treatments, cognitive-behavioural therapy (CBT) is the most commonly used..$^{20}$ The main form of the treatment is individual consultation. The typical length of the CBT treatment is several months and generally requires 8-28 treatments, ranging from 1 to 2 hours each time. The treatment content includes: (1) identification of cognitive distortions related to gaming behaviour; (2) looking for evidence that can confirm this cognitive distortion; (3) evaluating core beliefs and negative schema; (4) replacement with more adaptive thought patterns; (5) establishing relapse prevention and plan treatment steps; (6) dealing with self-control problems, and so forth. The results show that CBT is effective for people with gaming disorder and can change individuals' cognitions surrounding the game.

\section{Group therapy}

Psychological therapy, also known as team therapy or collective therapy, is conducted in groups or teams. The general framework for these kinds of therapy is 6-10 participants, one to two sessions a week (for 1-2 hours) for at least half a year. Treatment methods include lectures, activities and discussions. Treatment objectives are to reduce the patient's game dependence symptoms, promote the recovery of interpersonal relationships, improve the individual's self-confidence and manage the 
withdrawal from these games. ${ }^{21}$ The American Psychological Association believes that ${ }^{22}$ group therapy has certain advantages over individual therapy such as all participants have similar problems and face the same difficulties in life. By sharing game experiences with others, group participants can further recognise their own problems. In addition, group therapy can create a relatively closed and safe environment where sensitive topics about gaming disorder can be openly discussed. Because each person has different ways of dealing with their gaming disorder, group therapy can provide opportunities to learn from others who are dealing with gaming disorder, thereby improving their ability to cope.

\section{Family therapy}

Family therapy provides treatment by using psychological interventions in the family unit. It mainly includes traditional family therapy ${ }^{23}$ or marriage and family therapy. ${ }^{12}$ Often, a multilevel intervention model of drug abuse is applied, ${ }^{24}$ including family counselling and peer support groups. In addition to this, multifamily group therapy ${ }^{25}$ has been used to treat gaming disorder.

\section{Multimodal school-based group therapy}

It is a group psychotherapy method suitable for the school environment. It involves students, parents and teachers. Each group has 6-10 people. The purpose is to strengthen parent-child communication, promote family harmony, let parents recognise the problems of their children and find out their role in children's gaming behaviour as early as possible. Through psychological education, teachers also provide help for treatment. ${ }^{26}$

\section{Pharmacological treatment}

Those who advocate for the use of pharmacological treatment for gaming disorder are usually psychiatrists who believe gaming disorder is a mental disorder. ${ }^{12}$ The impulse shown by the person with gaming disorder towards the game has a similar neurobiological mechanism as the impulse of those addicted to substances towards their respective drug. ${ }^{9}$ Additionally, those with gaming disorder often have other comorbid mental disorders. ${ }^{27}$ This provides the foundation for pharmacological treatment.

Based on the points above, Dell'Osso and colleagues ${ }^{28}$ used escitalopram for the treatment of 19 adults with gaming disorder. In the first 10 weeks of drug treatment, the symptoms of game dependence improved in all patients. However, in the following 9 weeks of randomised double-blind controlled trials (half receiving drugs and half receiving placebos), there was no difference in the efficacy of the drug group and the control group. Bipeta and colleagues ${ }^{29}$ first treated 38 patients with simple obsessive-compulsive disorder who had gaming disorder or did not have gaming disorder with antianxiety therapy for 3 weeks and then treated with regular antidepressants (selective serotonin reuptake inhibitors or clomipramine) for 1 year. The results found that the patient's compulsive symptoms and game dependence were improved. Han and Renshaw ${ }^{30}$ used bupropion for the treatment of 50 patients with severe depression accompanied by excessive online gaming. The results showed that the patient's cravings for games were significantly reduced ${ }^{31}$ the time spent on the internet was shortened and the symptoms of depression were improved. After that, Han and colleagues ${ }^{32}$ used a central nervous stimulant, methylphenidate, to treat 62 children with attention deficit hyperactivity disorder (ADHD) who experienced excessive gaming. It was found that the degree of gaming disorder and the amount of time using the internet decreased significantly, and the symptoms of ADHD were also improved. In addition, there are also studies where the opioid receptor antagonist naltrexone was found to be effective against gaming disorder. ${ }^{33}$

The above limited data suggest that drug therapy (mostly antidepressants) can improve patients' game dependence symptoms, and can significantly shorten the time of internet use and reduce the cravings to play games. However, further research is needed to determine the efficacy, appropriate dose and course of treatment of psychotropic substances in gaming disorder. When the clinician uses drug treatment, he must closely observe the patient's condition and adjust the drug dose in time to prevent adverse reactions.

\section{Combined therapy}

Comprehensive treatment is an intervention that combines CBT with other treatment methods. It includes CBT combined with drug therapy, other forms of psychotherapy or physiotherapy.

\section{CBT combined drug treatment}

Kim and colleagues ${ }^{34}$ attempted to treat 65 adolescents with depressive disorder with amphetamine combined with CBT and found that it was effective for game dependence symptoms and depression. Santos and colleagues ${ }^{35}$ combined antidepressants and antianxiety drugs to treat gaming disorder. In this study, they found that patients' anxiety and gaming dependence symptoms were significantly improved.

\section{CBT combined motivation enhancement therapy}

According to motivation enhancement therapy's (MET) treatment experience with alcohol addiction, Poddar and colleagues $^{36}$ first tried this method for gaming disorder. This MET-CBT approach consists of a series of stages': (1) a contemplation stage (ie, initial sessions of rapport building, a detailed interview and case formulation $)^{2}$; (2) a preparation stage (ie, sessions delivered in an empathetic atmosphere to emphasise psychoeducation, including managing physiological and emotional arousal through relaxation techniques, and a cost-benefit analysis of game addiction); and $^{3}$ (3) a contract stage with the patient, the parents and the therapist (ie, behaviour modification of gaming, reducing time spent online and promoting healthy activities). ${ }^{19}$ After treatment, the 
patient's gaming time was significantly reduced and the learning performance was significantly improved.

\section{CBT combined electroacupuncture therapy}

There are people in China who tried to use this method and thought that the combined treatment group in improving game dependence symptoms is better than the individual psychotherapy group. However, the effectiveness and safety of the CBT combined electroacupuncture therapy of gaming disorder need to be verified. ${ }^{37}$

\section{PREVENTION OF GAMING DISORDER}

The causes of gaming disorder are complex, involving many biopsychosocial factors, and the treatment is relatively difficult. Therefore, prevention is even more important. The current preventive measures against gaming disorder are as follows:

\section{Limit the use of games}

In view of the potential addictive nature of computer and games, the long gaming time and the problem of overuse of the internet, limiting usage is a solution. ${ }^{38}$ Measures are: (1) prohibition of game access: the government requires game providers to prevent players from accessing their games for a specific period of time during the day; and (2) parental controls: parents control their children's computers through various measures, such as limiting game contents and time.

\section{Warning messages}

Some gaming companies have released in-game warning information related to the risks of excessive game playing. This information could be similar to the health warning information that appears on tobacco and alcohol packages. ${ }^{39}$ Based on the efficiency of cigarette warning labels ${ }^{40}$ it can be assumed that such warning information helps to raise awareness of the harmful consequences of excessive gaming. Király and colleagues suggest that customising the warnings in games depending on the time gamers spend on gaming. ${ }^{38}$ This strategy allows targeting problem behaviours specifically without affecting the non-problematic gamers' enjoyment of a largely healthy pastime activity. ${ }^{41}$

\section{Potential interventions}

In order to reduce the negative consequences of games, the government should promulgate relevant policies requiring all departments to provide appropriate help and treatment for people with gaming disorder. ${ }^{42}$ In the case of problematic gaming, the clinician is one of the firsts to identify the potential risks of gaming disorder and provide some help to game players. ${ }^{38}$ Since most gaming operators can collect players' data regarding the time spent on gaming, they could contact those gamers who play much more time than the average and offer them contact information for possible referral services. ${ }^{43}$ Game companies should also participate in actions to prevent and treat gaming disorder, such as reducing rewards and adding warnings in the game. ${ }^{44}$ Game providers should raise game prices and limit minors' premature contact with games. Governments should establish adequate prevention and treatment centres for game dependence, providing preventive education, counselling services and treatment interventions. ${ }^{45}$

\section{THE DEBATES AND FUTURE RESEARCH DIRECTIONS ON GAMING DISORDER}

Whether or not gaming disorder is a mental disorder is still controversial. However, most scholars believe that gaming disorder is an addiction-based mental disorder. ${ }^{11}$ First of all, gaming disorder has similar biological mechanism of substance use disorder and is related to the dopamine reward system at the edge of the middle brain. ${ }^{91146}$ When the player is playing, the level of dopamine neurotransmitters in the brain increases, which in turn produces pleasure. If this pleasure stimulates the brain repeatedly, the brain is rewarded, and the player will remember this feeling, resulting in addiction. ${ }^{47}$ Second, the response of the brain of people with gaming disorder to game-related clues may be similar to that seen in those with substance use disorder. ${ }^{48}$ Functional MRI results show that, compared with substance use disorder and other behavioural addictions (such as pathological gambling), gaming disorder may exhibit similar neurological activities in addictive brain regions (prefrontal cortex, nucleus accumbens septi, anterior cingulate area, caudal nucleus, and so on). ${ }^{4}$ Third, drug and psychological interventions can relieve the symptoms of people with gaming disorder and support their biological basis of biochemistry, cognition and behaviour. ${ }^{49}$ Finally, the genetic polymorphisms found in people with gaming disorder are related to substance use disorder and pathological gambling. The polymorphism of two genes related to substance use disorder (Taq1A1 allele of dopamine D2 receptor and Val158M and others allele in catecholamine-O-methyltransferase gene) has a higher incidence rate in gaming disorder. ${ }^{50}$ All of the above evidences suggest that gaming disorder is an addictive mental disease.

Opponents believe that: ${ }^{51}$ (1) although some manifestations of gaming disorder and substance-related disorder are similar, gaming disorder does not have the physical symptoms of substance use disorder. It is only manifested as a psychological dependence, so it is not an addictive behaviour. ${ }^{12}$ (2) Gaming disorder should be classified as an impulse control disorder, ${ }^{52}$ because persons with gaming disorder cannot control their own behaviour and the amount of time playing games. These persons engage in compulsive overplaying, spending so much time playing games that the results are addictive behaviour and loss in social functioning. (3) The functional damage caused by gaming has not been fully confirmed. (4) Gaming behaviour may be a coping mechanism to alleviate a disorder, not an independent disorder. (5) The inclusion of gaming disorder as a disease in ICD-11 may stigmatise some normal gamers and even lead to the possibility of 
excessive medical treatment. (6) Gaming disorder as a diagnostic type might be the result of moral panic. ${ }^{53}$

Up to now, many aspects of gaming disorder are still controversial. For instance, (1) whether or not gaming disorder is a mental illness; (2) the magnitude of the harm of gaming disorder; (3) the relationship between gaming disorder and other comorbid mental disorders; (4) the clinical manifestation of gaming disorder, the aetiology and the pathogenesis of gaming disorder. In addition, there are few studies on the positive effects of games. At present, the epidemiological survey data on gaming disorder among countries around the world are still in serious shortage. Therefore, future research directions include: (1) the epidemiological investigation of gaming disorder; (2) the development and standardisation of diagnostic tools; (3) the influencing factors of game behaviour; (4) brain imaging and neurobiology; and (5) treatment and prevention. In addiction, research on the positive role of gaming is also needed to clarify the nature of gaming disorder.Abstract translation 1

\section{Acknowledgements The authors thank the reviewers and editors of this article.}

Contributors Wang Qianjin: completed the summary, definition of gaming disorder, diagnosis, writing and full-text integration of treatment. Ren Honghong: document retrieval, writing and draft revision to complete gaming disorder prevention. Long Jiang: completed the adjustment of writing and writing format of gaming disorder epidemiology and aetiology. Liu Yueheng: completed the writing of the gaming disorder background and need to solve problems in the future.Liu Tieqiao: provided an outline of the article, guide writing, full-text revision and final draft.

Funding This work was supported by grants from the National Key R\&D Program of China(2017YFC1310400) and the National Natural Science Foundation of China(81371465 and 81671324). The sponsors have no role in the planning, conduct and publication of this work.

Competing interests All authors declare that they have no any conflict of interest in this article.

Patient consent for publication Not required.

Provenance and peer review Commissioned; externally peer reviewed.

Data availability statement № additional data are available.

Open access This is an open access article distributed in accordance with the Creative Commons Attribution Non Commercial (CC BY-NC 4.0) license, which permits others to distribute, remix, adapt, build upon this work non-commercially, and license their derivative works on different terms, provided the original work is properly cited, appropriate credit is given, any changes made indicated, and the use is non-commercial. See: http://creativecommons.org/licenses/by-nc/4.0/.

\section{REFERENCES}

1 World Health Organization. Gaming Disorder - What are gaming disorder? 2018. Available: https://www.who.int/features/qa/gamingdisorder/zh/

2 China Internet Network Information Center (CNNIC). The 42nd statistical report on the development of China's Internet, 2018. Available: http://www.cnnic.net.cn/hlwfzyj/hlwxzbg/hlwtjbg/201808/ t20180820_70488.htm

3 Mihara S, Higuchi S. Cross-sectional and longitudinal epidemiological studies of Internet gaming disorder: a systematic review of the literature. Psychiatry Clin Neurosci 2017;71:425-44.

4 Long J, Liu T, Liu Y, et al. Prevalence and correlates of problematic online gaming: a systematic review of the evidence published in Chinese. Curr Addict Rep 2018;5:359-71.

5 Przybylski AK, Weinstein N, Murayama K. Internet gaming disorder: investigating the clinical relevance of a new phenomenon. AJP 2017;174:230-6.

6 Rehbein F, Kliem S, Baier D, et al. Prevalence of Internet gaming disorder in German adolescents: diagnostic contribution of the nine
DSM- 5 criteria in a state-wide representative sample. Addiction 2015; $110: 842-51$

7 Wikipedia. Video game addiction, 2018. Available: https://en. wikipedia.org/wiki/Video_game_addiction

$8 \mathrm{Mez}$ B. A quiet killer: why video games are so addictive, 2013. Available: https://thenextweb.com/insider/2013/01/12/what-makesgames-so-addictive/

9 Fauth-Bühler M, Mann K. Neurobiological correlates of Internet gaming disorder: similarities to pathological gambling. Addictive Behaviors 2017;64:349-56.

10 Kornhuber J, Zenses E-M, Lenz B, et al. Low 2D:4D values are associated with video game addiction. PLoS ONE 2013;8:e79539.

11 Saunders JB, Hao W, Long J, et al. Gaming disorder: its delineation as an important condition for diagnosis, management, and prevention. Journal of Behavioral Addictions 2017;6:271-9.

12 Dong GH. Internet addiction disorder[M]//Lu L. Shen YuCun's Psychiatry. 6th edn. Chinese: Beijing: People's Medical Publishing House (PMPH), 2018: 691

13 Petry NM, Rehbein F, Ko C-H, et al. Internet gaming disorder in the DSM-5. Curr Psychiatry Rep 2015;17.

14 Scutti S. WHO classifies 'gaming disorder'as mental health condition[J]. CNN 2018;27.

15 Aarseth E, Bean AM, Boonen H, et al. Scholars' open debate paper on the World Health Organization ICD-11 Gaming Disorder proposal. Journal of Behavioral Addictions 2017;6:267-70.

16 Chakraborty K, Basu D, Vijaya Kumar KG. Internet addiction: consensus, controversies, and the way ahead. East Asian Arch Psychiatry 2010;20:123-32.

17 Zhong N, Du J, Vladimir P, et al. Research progress of game disorder and controversy as a new diagnostic classification of ICD-11 mental and behavioral disorder (draft). Chinese Journal of Psychiatry 2018;51:149-52.

18 Hao W, Zhao M, Li J. Theory and practice of addiction medicine. Beijing: People's Medical Publishing House (PMPH), 2016: 238-95.

19 Kuss DJ, Lopez-Fernandez O. Internet addiction and problematic internet use: a systematic review of clinical research. WJP 2016;6.

20 Stevens MWR, King DL, Dorstyn D, et al. Cognitive-behavioral therapy for Internet gaming disorder: a systematic review and metaanalysis. Clin Psychol Psychother 2018.

21 Fan FM. Group psychotherapy[M]//Lu L. Shen YuCun's Psychiatry. 6th edn. Chinese, Beijing: People's Medical Publishing House (PMPH), 2018: 816.

22 American Psychological Association. Psychotherapy: understanding group therapy. American Psychological Association, 2015.

23 Tai YP, Kim S, Lee J. Family therapy for an Internet-addicted young adult with interpersonal problems. J Fam Ther 2014;36:394-419.

24 Shek DT, Tang V M LCY. Evaluation of an Internet addiction treatment program for Chinese adolescents in Hong Kong. Adolescence 2009;44:359-73.

25 Liu Q-X, Fang X-Y, Yan N, et al. Multi-family group therapy for adolescent Internet addiction: exploring the underlying mechanisms. Addictive Behaviors 2015;42:1-8.

26 Du Y S JW, Vance A. Longer term effect of randomized, controlled group cognitive behavioural therapy for Internet addiction in adolescent students in Shanghai. Aust N Z J Psychiatry 2010;22:129-34.

27 González-Bueso V, Santamaría J, Fernández D, et al. Association between Internet gaming disorder or pathological video-game use and comorbid psychopathology: a comprehensive review. IJERPH 2018;15.

28 Dell'Osso B, Hadley S, Allen A, et al. Escitalopram in the treatment of impulsive-compulsive Internet usage disorder: an open-label trial followed by a double-blind discontinuation phase. $J$ Clin Psychiatry 2008;69:452-6.

29 Bipeta R, Yerramilli SS, Karredla AR, et al. Diagnostic stability of Internet addiction in obsessive-compulsive disorder: data from a naturalistic one-year treatment study. Innov Clin Neurosci 2015;12:14-23

30 Han DH, Renshaw PF. Bupropion in the treatment of problematic online game play in patients with major depressive disorder. $J$ Psychopharmacol 2012;26:689-96.

31 Doug Hyun H, Jun Won H, Renshaw PF. Bupropion sustained release treatment decreases craving for video games and cue-induced brain activity in patients with Internet video game addiction. Exp Clin Psychopharmacol 2010;18.

32 Han DH, Lee YS, Na C, et al. The effect of methylphenidate on internet video game play in children with attention-deficit/ hyperactivity disorder. Comprehensive Psychiatry 2009;50:251-6.

33 Bostwick JM, Bucci JA. Internet sex addiction treated with naltrexone. Mayo Clinic Proceedings 2008;83:226-30. 
34 Kim SM, Han DH, Lee YS, et al. Combined cognitive behavioral therapy and bupropion for the treatment of problematic on-line game play in adolescents with major depressive disorder. Computers in Human Behavior 2012;28:1954-9.

35 Santos V, Nardi A, King A. Treatment of Internet addiction in patient with panic disorder and obsessive compulsive disorder: a case report. CNS Neurol Disord Drug Targets 2015;14:341-4.

36 Poddar S, Sayeed N, Mitra S. Internet gaming disorder: application of motivational enhancement therapy principles in treatment. Indian $J$ Psychiatry 2015;57.

37 Zhu TM, Jin RJ, Zhong XM, et al. Effects of electroacupuncture combined with psychologic interference on anxiety state and serum $\mathrm{Ne}$ content in the patient of Internet addiction disorder. Zhongguo Zhen Jiu 2008;28.

38 Király O, Griffiths MD, King DL, et al. Policy responses to problematic video game use: a systematic review of current measures and future possibilities. Journal of Behavioral Addictions 2018;7:503-17.

39 Van Rooij AJ, Meerkerk G-J, Schoenmakers TM, et al. Video game addiction and social responsibility. Addiction Research \& Theory 2010;18:489-93.

40 Azagba S, Sharaf MF. The effect of graphic cigarette warning labels on smoking behavior: evidence from the Canadian experience. Nicotine \& Tobacco Research 2013;15:708-17.

41 Billieux J, Schimmenti A, Khazaal Y, et al. Are we overpathologizing everyday life? A tenable blueprint for behavioral addiction research. Journal of Behavioral Addictions 2015;4:119-23.

42 Policy KDJ. Prevention, and regulation for Internet gaming disorder. J Behav Addict 2018;7:553-5.

43 Auer MM, Griffiths MD. Testing normative and self-appraisal feedback in an online slot-machine pop-up in A real-world setting. Front. Psychol. 2015;6.
44 Yousafzai S, Hussain Z, Griffiths M. Social responsibility in online videogaming: what should the videogame industry do? Addiction Research \& Theory 2014;22:181-5.

45 Dau W, Hoffmann JDG, Banger M. Therapeutic Interventions in the Treatment of Problematic Internet Use-Experiences from Germany[M]//Internet addiction. Springer, Cham 2015:183-217.

46 Dong G, Li H, Wang L, et al. Cognitive control and reward/loss processing in Internet gaming disorder: results from a comparison with recreational Internet game-users. European Psychiatry 2017;44:30-8.

47 Mitchell P. Internet addiction: genuine diagnosis or not? The Lancet 2000;355.

48 Liu L, Yip SW, Zhang J-T, et al. Activation of the ventral and dorsal striatum during cue reactivity in Internet gaming disorder. Addiction Biology 2017;22:791-801.

49 C H K, Liu GC, Hsiao S, et al. Brain activities associated with gaming urge of online gaming addiction. J Psychiatr Res 2009;43:739-47.

50 Han DH, Lee YS, Yang KC, et al. Dopamine genes and reward dependence in adolescents with excessive Internet video game play. Journal of Addiction Medicine 2007;1:133-8.

51 Starcevic V, Aboujaoude E, Disorder IG, et al. Obsessive-compulsive disorder. and Addiction 2017;4:317-22.

52 Su W, Fang X, Miller JK, et al. Internet-based intervention for the treatment of online addiction for college students in China: a pilot study of the healthy online Self-Helping center. Cyberpsychology, Behavior, and Social Networking 2011;14:497-503.

53 Rumpf $\mathrm{H}-\mathrm{J}$, Achab S, Billieux J, et al. Including gaming disorder in the ICD-11: the need to do so from a clinical and public health perspective. J Behav Addict 2018;7:556-61.

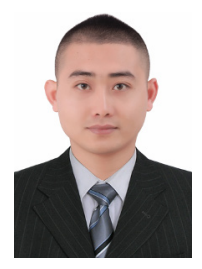

Qianjin Wang obtained a bachelor's degree in clinical medicine in 2015 from Jining Medical College. He is currently studying for a master's degree in psychiatry at Institute of Mental Health, The Second Xiangya Hospital of Central South University. His research interest is addiction medicine. 